\title{
EDITORIAL Prospects for RNA delivery with nanotechnologies
}

\author{
Gene Therapy (2017) 24, 121; doi:10.1038/gt.2017.15
}

This issue of Gene Therapy focuses on recent developments and promising applications in nanoparticle delivery formulations. Gene therapy with viral vectors has achieved some notable successes in recent years, for example, in the development of treatments for diseases such as immunodeficiencies, haemoglobinopathies, clotting disorders and retinal degeneration, with many other exciting developments in cancer with engineered T cells. Gene therapy products are now finding their way to the market for treatment of rare diseases, but most of this activity is focused on viral vectors. Non-viral vectors, as alternatives to viral vectors, offer advantages of safety and larger packaging capacity, but clinical trials in diseases where liposomal vectors seemed particularly beneficial, such as lung disease of cystic fibrosis, have highlighted problems of efficiency and duration of gene expression. The most promising areas of clinical activity with non-viral nanoparticles are currently limited to small interfering RNA (siRNA) delivery. However, recent developments in nucleic acid technologies including siRNA and oligonucleotides but also chemically modified messenger RNA (mRNA) and minicircle DNA, for which viral vectors are not an option, have led to a resurgence of interest in non-viral methods of delivery and nanoparticles in particular. Nanoparticles, as seen in the majority of articles in this issue, are rapidly emerging as de facto RNA delivery agents, particularly, for mRNA (Guan and Rosenecker), siRNA (Jones and Merkel; Scherman et al.; Schiffelers et al.) and RNA-based gene editing formulations (Wang et al.).

The burgeoning field of CRISPR/Cas9 gene editing technologies also offers a range of new opportunities for nanoparticles that play to the strengths of these adaptable delivery systems as described in the article by Wang et al. in this issue. Formulations based on Cas9 mRNA and synthetic guide RNA, or preassembled Cas9 ribonucleoprotein will require nanoparticle formulations and methodologies for delivery. The targeting of genetic loci with Cas9 by guide RNA and the introduction of double-strand breaks in the genome occur rapidly after transfection making long-term expression redundant, and in fact, it is even thought to be undesirable to have persistent expression of Cas9 to limit offtarget effects in the genome. Thus, transient expression, characteristic of nanoparticle transfection, once regarded as a limitation, may, in the context of gene editing, prove to be a strength in terms of specificity and efficiency. A further great advantage is that even if a single dose does not achieve the desired level of gene disruption or repair, the low level of immunogenicity of most nanoparticle formulations means that repeated dosing may be performed until appropriate levels of gene modification are achieved. Such an approach would likely be challenging with viral vectors due to the adaptive immune system eradicating transduced cells.

This increasing demand for nanoparticles will also likely lead to a better understanding of their optimal composition, structure and biological interactions with cells. The widespread use of just two viral vectors, where with a few exceptions lentivirus are used for ex vivo cell therapy and adeno-associated virus (AAV) for in vivo gene therapy, has undoubtedly benefited their rapid development and uptake for therapeutic uses. Nanoparticles, on the other hand, although generally limited to lipid or polymeric formulations, are often bespoke formulations to each laboratory. In this issue, a wide range of formulations are described including emerging technologies such as graphene nanoparticles (Vincent et al.) and exosomes (Schiffelers et al.), as well as liposomal and polymeric formulations. Novel nanoparticles are still required for nucleic acid delivery that will be important to characterise for their chemical and biophysical properties before clinical use. This requirement for multidisciplinary approaches in addition to the efficacy of viruses may have retarded the development of nanotechnologies so far, but with the emergence of nucleic acids and therapeutic strategies that require their use, such studies acquire a new urgency.

Nanoparticles offer a wide range of applications in vivo, and may be targeted by ligands or by their innate targeting properties for organs such as the liver where nanoparticles are most advanced in their clinical development, although other targets such as inflammatory joints may be targeted systemically while the eye can be targeted by direct delivery routes (Scherman et al.). A great deal of nanoparticle development is targeted towards cancer therapies, and an example of that is described by Jones and Merkel in this issue in developing siRNA therapies for breast cancer. A challenge here is enabling the nanoparticle to avoid the liver after systemic delivery, since many such formulations naturally accumulate in highly vascularised organs including liver, lung and spleen, reducing tumour targeting capacity.

Despite the successes with viral vectors, challenges remain including overcoming the innate and adaptive immune responses to viruses and concerns over random integration leading to insertional oncogenesis with lentiviral vectors, while arguments over links between AAV and liver cancer persist. Nanoparticles offer the opportunity to overcome such limitations, provided their efficiency levels are sufficient and moreover expand the usefulness of new nucleic acid technologies in developing therapeutics. The reviews in this issue on new nanoparticle formulations, approaches to nucleic acid delivery and therapeutic strategies are therefore a timely reminder of the potential of such systems.

\section{CONFLICT OF INTEREST}

The author declares no conflict of interest.

SL Hart

Experimental and Personalised Medicine Section, Genetics and Genomic Medicine Programme, UCL Great Ormond Street Institute of Child Health, University of London Institute of Child Health Genetics and Genomic Medicine, London, UK E-mail: s.hart@ucl.ac.uk 